\title{
PROCESAMIENTO DE LA INFORMACIÓN DE AMENAZA FÍSICA EN LA HIPOCONDRÍA: UN ESTUDIO EXPLORATORIO UTILIZANDO EL PARADIGMA DE STROOP
}

\author{
M. PILAR MARTÍNEZ ${ }^{1}$ Y AMPARO BELLOCH ${ }^{2}$ \\ ${ }^{1}$ Universidad de Granada \\ ${ }^{2}$ Universidad de Valencia
}

(Recibido el 22 de septiembre de 1997)

\begin{abstract}
Desde una perspectiva cognitiva se plantea que los pacientes con trastornos emocionales presentan un sesgo atencional que favorece el procesamiento selectivo de la información relacionada con el problema que les afecta. Uno de los procedimientos más utilizados para explorar este tipo de sesgos es el paradigma de Stroop. En el presente estudio se analizó el procesamiento selectivo de la información de amenaza física en sujetos hipocondríacos mediante este paradigma. La muestra estuvo formada рог 17 pacientes con hipocondría, 17 pacientes con trastorno por angustia y 17 sujetos normales. Todos ellos cumplimentaron las cinco tarjetas de las que constaba la versión elaborada de la tarea de Stroop (palabras de "oes", palabras neutras, palabras de amenaza social, palabras de amenaza física, y palabras de colores), así como diversos cuestionarios (Inventario de Ansiedad Estado-Rasgo, Inventario de Depresión de Beck, Escala de Hipocondríasis del Inventario de Personalidad Multifásico de Minnesota, Escalas de Actitud hacia la Enfermedad, y Cuestionario de Conducta de Enfermedad). Se constató la existencia de un sesgo atencional específico referido a las amenazas físicas en los pacientes con hipocondría y en los pacientes con trastorno por angustia. Asimismo, se observó una relación entre el sesgo atencional hacia las amenazas físicas y las medidas que evalúan las preocupaciones hipocondríacas.
\end{abstract}

Palabras clave: Hipocondría, amenaza física, sesgo atencional, procesamiento selectivo, tarea de Stroop.

\section{Information processing of physical threat in hypochondriasis: an exploratory study using the Stroop paradigm}

The cognitive approaches had proposed that patients with emotional disorders show an attentional bias that favours the selective processing of information related to their problem. One of the most used procedures to explore this kind of bias is the Stroop paradigm. In the present study the selective processing of the physical threat information in hypochondriacal subjects was explored using the Stroop paradigm. The subjects were 17 hypochondriac patients, 17 panic disorder patients, and 17. normal subjects. All subjects fulfilled the five cards composing the elaborated version of the Stroop test ("os" words, neutral words, social threat words, physical threat words, and colour words), and several questionnaires (State-Trait Anxiety Inventory, Beck's Depression Inventory, Hypochondriasis Scale of Minnesota Multiphasic Personality Inventory, Illness Attitude Scales, and Illness Behaviour Questionnaire). The specific attentional bias related to physical threat in hypochondriasis patients and panic disorder patients was confirmed. Also, the relationship between the atentional bias to physical threat and the measures that evaluate hypochondriacal concerns was observed.

Key words: Hypochondriasis, physical threat, attentional bias, selective processing, Stroop test.

Agradecimientos: Este estudio ha sido realizado gracias a una beca de investigación concedida por la Conselleria de Cultura, Educació i Ciència de la Generalitat Valenciana para llevar a cabo una investigación más amplia de la que forma parte este trabajo.
Correspondencia: M. Pilar Martínez, Departamento de Personalidad, Evaluación y Tratamiento Psicológicos. Facultad de Psicología. Universidad de Granada. Campus Universitario de Cartuja, 18071, Granada. Telef.: 958244 251. Fax: 958243 749. E-Mail: mnarvaez@platon.ugr.es. 


\section{INTRODUCCIÓN}

Desde hace algunos años los psicopatólogos de orientación cognitiva vienen interesándose por el estudio de los sesgos cognitivos que presentan los pacientes con trastornos emocionales. Una de las hipótesis más sólidas que se han formulado sostiene que estos pacientes procesan selectivamente la información que posee un contenido congruente con su problemática. La presencia de tales disfunciones ha sido analizada en diversas condiciones clínicas tales como el trastorno por angustia (McNally, Riemann y Kim, 1990), la fobia social (Hope, Rapee, Heimberg y Dombeck, 1990), el trastorno obsesivo-compulsivo (Foa, Ilai, McCarthy, Shoyer y Murdock, 1993), la depresión mayor (Carter, Maddock y Magliozzi, 1992), etc.

Recientemente, los trastornos somatoformes, y en concreto la hipocondría, han empezado a formar parte del abanico de trastornos conceptualizados desde los modelos cognitivos. Las formulaciones más consistentes sobre el desarrollo de la hipocondría son, por una parte, aquella que considera que la característica central de los pacientes que presentan este trastorno es su tendencia a amplificar las sensaciones corporales benignas (Barsky, 1992), $y$, por otra parte, aquella que erige como elemento principal su propensión a interpretar de manera catastrófica dichas sensaciones (Warwick y Salkovskis, 1990). Tomando como referencia estos y otros planteamientos, en los últimos años se han realizado algunos estudios destinados a identificar los déficits en estructuras y procesos cognitivos que pudieran ser los responsables de la fenomenología de este trastorno. Los elementos cognitivos más estudiados han sido la inclinación a detectar, atender y percibir las sensaciones corporales (sesgos atencionales); la proclividad a interpretar tales sensaciones como señal de enfer- medad (sesgos interpretativos); y la tendencia a recordar hechos relacionados con la enfermedad (sesgos mnésicos).

Centrándonos en los sesgos atencionales, algunos estudios (Cioffi, 1991; Pauli, Schwenzer, Brody, Rau y Birbaumer, 1993; Schmidt, Wolfs-Takens, Oosterlaan y Van den Hout, 1994) han puesto de manifiesto que los pacientes hipocondríacos muestran una atención selectiva hacia la información vinculada a su preocupación por la salud. Por ejemplo, Cioffi (1991) estudió el modo en que la atención focalizada en las sensaciones corporales influye en el significado que se asigna a éstas, encontrando que son las situaciones en las que se combinan la auto-observación corporal y la anticipación de peligro donde se produce una interpretación negativa de las sensaciones corporales. Pauli et al. (1993) estudiaron los sesgos atencionales y la sensibilidad al dolor y hallaron que los sujetos hipocondríacos reducían su ejecución en un test de concentración-rendimiento mientras permanecían conectados a un estimulador de dolor, incluso cuando se les informaba de que no recibirían el estímulo doloroso. Schmidt et al. (1994) estudiaron la relación entre la atención y las expectativas y la percepción de la sintomatología interoceptiva, encontrando que ambos factores, tanto combinados como por separado, influyen en el informe de síntomas físicos.

Al margen de estos trabajos, y de acuerdo con la bibliografía revisada, no existe ningún estudio que haya investigado la atención diferencial a los estímulos amenazantes en la hipocondría a través de uno de los procedimientos para lograr este fin: la tarea de Stroop. La versión original de esta prueba (Stroop, 1935) consiste en presentar al sujeto una serie de tarjetas que contienen palabras de nombres de colores, y pedirle que, ignorando el significado de las palabras, nombre el color en que están impresas 
con la mayor rapidez y exactitud posible. La versión «emocional» de la tarea de Stroop difiere de la inicial en que incluye palabras de contenido emocional. En esta prueba se considera que el sesgo atencional hacia ciertos temas se refleja en una mayor lentitud al nombrar las palabras que poseen un contenido amenazante para el sujeto. Este efecto es debido a que el significado de la palabra y el color con que está escrita compiten para captar los mismos recursos atencionales disponibles (McNally et al., 1990).

En el presente trabajo nos propusimos estudiar si los pacientes hipocondríacos presentan una tendencia a analizar preferentemente la información coherente con el núcleo de su problema. Los objetivos concretos que nos planteamos fueron los siguientes: 1) estudiar si los pacientes hipocondríacos muestran un sesgo específico en el procesamiento de la información de amenaza física; 2) explorar las diferencias existentes entre los pacientes hipocondríacos y otros sujetos (pacientes con trastorno por angustia y controles normales) en el procesamiento de la información neutral y la amenazante (física y social); y 3) analizar las relaciones que se establecen entre el procesamiento sesgado de información de diferente contenido temático (neutral, amenaza física y amenaza social) y diversas medidas clínicas (ansiedad, depresión, síntomas somáticos, actitudes y conductas de enfermedad).

\section{MÉTODO}

\section{Muestra}

La muestra estuvo compuesta por dos grupos psicopatológicos y uno normal. Los dos primeros fueron 17 pacientes con hipocondría (13 mujeres y 4 hombres; 20 50 años) y 1.7 pacientes con trastorno por angustia con y sin agorafobia (12 mujeres y 5 hombres; 18-49 años). En ambos casos el diagnóstico se estableció de acuerdo con los criterios del DSM-III-R (APA, 1987) ya que en el momento de la realización del estudio no se disponía de la versión española definitiva del DSMIV. El grupo normal estuvo constituido por 17 sujetos que no presentaban problemas psicológicos (13 mujeres y 4 hombres; 21-51 años). Se contemplaron los siguientes criterios de exclusión en los tres grupos: edad inferior a 18 años o superior a 65 años, historia de adicción a drogas o alcoholismo, y presencia actual de enfermedad física grave diagnosticada. Todos los participantes fueron igualados en diversas características sociodemográficas tales como sexo y edad, $F(2,48)=0,65$. No obstante, los grupos difirieron en cuanto al nivel de instrucción, $\left(\chi^{2}(2)=6,46, p<0,05\right.$, siendo la única diferencia significativa la correspondiente al contraste entre el grupo de hipocondría y el grupo de trastorno por angustia (K-S $=1,37, p<0,05)^{1}$ (el primer grupo presentó un nivel de instrucción inferior al segundo).

\section{Instrumentos}

Para establecer el diagnóstico en los grupos clínicos se utilizó una versión modificada de la Entrevista Diagnóstica Estructurada para la Hipocondría (Barsky, Cleary, Wyshak, Spitzer, Williams y Klerman, 1992) que incluyó los criterios del DSM-III-R, y la Entrevista Clínica Estructurada para el DSM-III-R (sección del trastorno por angustia) (Spitzer, Williams, Gibbon y First, 1990).

Inventario de Ansiedad Estado-Rasgo, STAI (Spielberger, Gorsuch y Lushene, 1970). Incluye dos escalas independien-

\footnotetext{
'Para la variable edad se realizó un análisis de varianza de un factor; para la variable nivel de instrucción se calcularon las pruebas de Kruskal-Wallis y de Kolmogorov-Smirnov.
} 
tes que evalúan aspectos distintos de la ansiedad: como estado (E) y como rasgo (R). Cada escala consta de 20 enunciados que son puntuados según un intervalo que va de 0 ("nada» en el STAI-E; "casi nunca» en el STAI-R) a 3 ("mucho» en el STAI-E; "casi siempre» en el STAI-R).

Inventario de Depresión de Beck, BDI (Beck, Rush, Shaw y Emery, 1979). Comprende 21 grupos de 4 enunciados cada uno que describen sintomatología depresiva. La puntuación de los ítems abarca entre 0 y 3.

Escala de Hipocondríasis (Hs) del Inventario de Personalidad Multifásico de Minnesota, MMPI (Hathaway y McKinley, 1967). Está formada por 33 preguntas de respuesta dicotómica («verdadero" o «falso") que miden la preocupación anormal por las propias funciones corporales. En el presente estudio esta escala ha sida utilizada como un listado de síntomas somáticos.

Escalas de Actitud hacia la Enfermedad, IAS (Kellner, 1986). Evalúan las características psicopatológicas vinculadas a la hipocondría y a la conducta anormal de enfermedad. Comprenden 29 ítems (agrupados en 9 escalas) a los que se contesta (salvo en dos de ellos) de acuerdo con una escala que se extiende desde 0 («no») hasta 4 («casi siempre»). En este trabajo hemos utilizado la puntuación total de este instrumento.

Cuestionario de Conducta de Enfermedad, IBQ (Pilowsky y Spence, 1983). Estima las actitudes que sugieren formas inadecuadas de responder al propio estado de salud. Incluye 62 ítems (conforman 7 factores) a los que se responde según dos alternativas de respuesta ( $« \mathrm{si}$ » $\mathrm{o}$ «no»). En el presente trabajo hemos utilizado la puntuación total de esta medida.

Tarea de Stroop. La versión empleada en este estudio consta de 5 tarjetas de tamaño DIN A-4 de color blanco. Para cada una de ellas se seleccionó un grupo de 12 palabras que fueron escritas en 8 columnas de 12 palabras cada una (96 palabras por tarjeta). El grupo de palabras escogidas recibió una distribución aleatoria en cada columna de la tarjeta. Todas las palabras tuvieron un tamaño aproximadamente de $0,5 \mathrm{~cm}$. y fueron escritas en diferentes colores (rojo, azul, verde y negro). En todas las tarjetas los colores fueron asignados al azar a las 96 palabras, evitando que un color apareciese más de dos veces consecutivas en cada columna. Las palabras de todas las tarjetas, a excepción de la tarjeta de palabras de colores, fueron igualadas respecto a su frecuencia media de uso en el idioma español (se excluyeron tanto las palabras muy inusuales como las muy comunes), número de letras (entre 5 y 8) y parte variable de la oración (nombre, adjetivo $\mathrm{y}$ verbo). Las palabras fueron seleccionadas atendiendo a criterios racionales y a partir de la información recogida en la literatura publicada sobre la hipocondría y el trastorno por angustia. Un grupo de clínicos expertos valoró la idoneidad de las palabras. Las tarjetas empleadas fueron las siguientes:

1) Tarjeta de "oes». Cada ítem consistió en una serie de entre 5 y 8 "oes".

2) Tarjeta de palabras de colores. Los ítems fueron palabras de colores (rojo, azul, verde y negro) escritas en colores disonantes.

3) Tarjeta de palabras neutras. Los ftems estuvieron formados por palabras de contenido emocional neutro (p. ej., número, puerta, cuadro, escuchar).

4) Tarjeta de palabras de amenaza social. Se utilizaron palabras cuyo significado estuvo asociado a un posible daño o perjuicio procedente del contexto de las relaciones interpersonales (p. ej., estúpido, inepto, odiar, crítica).

5) Tarjeta de palabras de amenaza física. Los ítems fueron palabras con un contenido potencialmente relacionado con un daño o lesión a nivel físico (p. ej., tumor, cáncer, operar, enfermo). 
Como instrucción general, se solicitó a los sujetos que nombraran en voz alta el color en que estaban escritas las palabras y lo hicieran columna a columna hasta completar la tarjeta. Con el fin de controlar los efectos de la práctica se presentaron las tarjetas en orden aleatorio para cada sujeto.

Las mediciones que se efectuaron fueron: 1) índice de velocidad, que se extrae dividiendo el número de palabras que componen cada tarjeta (96) entre el tiempo (segundos) empleado en completar cada una de ellas; 2) índice de interferencia, resultante de la diferencia entre el índice de velocidad de la tarjeta de palabras neutras y el índice de velocidad de la tarjeta de palabras de amenaza física (o amenaza social); y 3) indice de contenido, que se obtiene de la diferencia entre el índice de velocidad de la tarjeta de palabras de amenaza física y el índice de velocidad de la tarjeta de palabras de amenaza social.

\section{Procedimiento}

Las muestras clínicas fueron recogidas en diversas Unidades de Salud Mental de Valencia (Burjassot, Massamagrell y Puçol) y Castellón (Vila-Real). La primera fase del estudio consistió en la evaluación efectuada por el psiquiatra o el psicólogo clínico de la unidad. Del grupo inicial de pacientes evaluados, los que exhibían preocupaciones hipocondríacas o ataques de pánico pasaron a una segunda fase en la que se realizó una entrevista de screening. Seguidamente, aquellos que reunían los criterios diagnósticos del DSM-III-R para la hipocondría o para el trastorno por angustia así como los requisitos adicionales establecidos, fueron seleccionados para la cumplimentación de varios cuestionarios y tareas experimentales. A los sujetos se les explicó que dichos instrumentos eran un método para profundizar en el conocimiento de su problemática. La muestra normal, al estar formada por personas que no presentaban trastornos psicológicos, fue recabada en contextos no asistenciales. A todas ellas se les dijo que el estudio pretendía explorar su opinión sobre la salud y la enfermedad.

\section{Análisis estadísticos}

Para analizar si los grupos de sujetos diferían en el índice de velocidad de las tarjetas que componían la tarea de Stroop se realizó un análisis de varianza (ANOVA) de medidas repetidas 3 (grupos) $x 5$ (tarjetas), siendo el primer factor «inter» y el segundo «intra». Asimismo, para los índices de interferencia y de contenido se calculó un ANOVA de medidas repetidas 3 (grupos) $\times 2$ (tarjetas), siendo de nuevo el primer factor «inter» y el segundo «intra». Con anterioridad a los ANOVAs se comprobó la homogeneidad de las varianzas mediante la prueba de Bartlett. Para explorar la existencia de diferencias entre las muestras en cada uno de los índices de las distintas tarjetas, se realizó un ANOVA de un factor (previo cálculo de la prueba de Levene para la homogeneidad de las varianzas), y a continuación se utilizó la prueba de Scheffé como contraste a posteriori. Con el objeto de determinar para cada grupo las diferencias en la velocidad en completar las láminas, se calculó la prueba $t$ de Student para muestras relacionadas. Finalmente, se calculó el coeficiente de correlación de Pearson para estudiar la relación entre los índices de velocidad y de interferencia de la prueba de Stroop y las diversas medidas clínicas consideradas. Para el análisis correlacional se utilizó conjuntamente los datos de los tres grupos de estudio. Todos los análisis estadísticos realizados fueron bilaterales y en ellos se tomaron como niveles 
de significación probabilidades inferiores $o$ iguales a 0,05 .

\section{RESULTADOS}

\section{Indices de velocidad}

En la Figura 1 se presenta la media de la latencia de respuesta (tiempo expresado en segundos) de los tres grupos en cada una de las tarjetas de la tarea de Stroop.

El ANOVA de medidas repetidas con las cinco condiciones de la prueba experimental, mostró un efecto principal significativo de los factores grupo, $F(2,48)=$ $5,07, p<0,01$, y tarjeta, $F(4,192)=116,01$, $p<0,005$; sin embargo, no resultó significativa la interacción grupo $\mathrm{x}$ tarjeta, $F(8,192)=1,81$. Cuando se trató de controlar la posible influencia del nivel de instrucción de los sujetos mediante un ANOVA de medidas repetidas con una covariante constante, el factor grupo siguió resultando significativo, $F(2,47)=$ $3,37, p<0,05$. Para determinar las diferencias entre los grupos en los índices de velocidad de las cinco condiciones, calculamos ANOVAs de un factor. No se observaron diferencias significativas entre los grupos en la velocidad con que completaron la tarjeta compuesta por "oes», $F(2,48)=1,68$; en cambio, si las hubo en la rapidez con que nombraron los colores de las palabras neutras, $F(2,48)=3,69, p<0,05$, de amenaza social, $F(2,48)=3,42, p<0,05$, de amenaza física, $F(2,48)=9,66, \quad p<0,005$, y de colores, $F(2,48)=4,65, p<0,05$. En concreto, la prueba post-hoc de Scheffé mostró que los sujetos hipocondríacos fueron más lentos que los sujetos normales en completar todas las tarjetas (a excepción de la de «oes»). Sin embargo, los pacientes con trastorno por angustia únicamente presentaron una tasa de velocidad menor que los sujetos normales en la condición

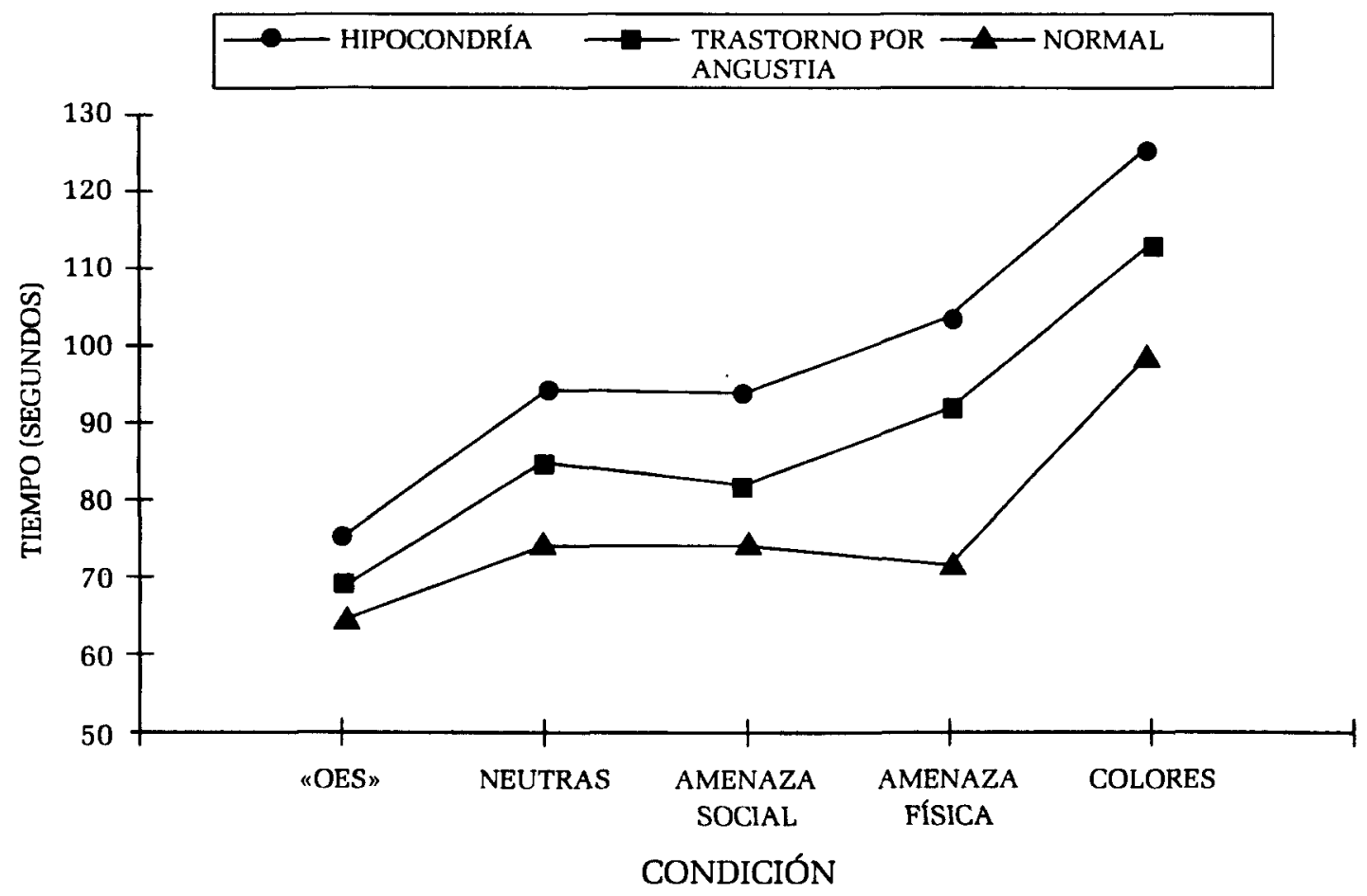

Figura 1. Tiempo medio tardado por cada grupo en completar las tarjetas de la tarea de Stroop. 
de Amenaza Física. Estos resultados se pueden consultar en la Tabla 1.

En cuanto a la rapidez con que cada grupo nombró los colores de las distintas condiciones (análisis intra-grupo), cabe señalar que no se apreciaron diferencias significativas entre los índices de velocidad de las tarjetas Neutra y de Amenaza Social en ninguno de ellos. En cambio, las diferencias entre los índices de velocidad de las tarjetas Neutra y de Amenaza Física si alcanzaron significación estadística en los grupos de hipocondría, $t=2,55$, $p(0,05, y$ trastorno por angustia, $t(16)=$ $2,10, p(0,05$. Asimismo, se detectaron en estos grupos diferencias significativas entre los índices de velocidad de las tarjetas de Amenaza Social y de Amenaza Física (hipocondría: $t(16)=3,04, p<0,01$; trastorno por angustia: $t(16)=3,82, p<0,005$. Estos resultados se presentan en la Tabla 2.

Indices de interferencia y de contenido

El ANOVA de medidas repetidas reali- zado con el índice de interferencia para la condición de palabras de Amenaza Social, mostró un efecto principal significativo para el factor grupo, $F(2,48)=3,92, p(0,05$, no alcanzando significación estadística los efectos del factor tarjeta, $F(1,48)=0,25$, ni de la interacción de ambos, $F(2,48)=0,38$. El factor grupo dejo de ser significativo $F(2,47)=2,32$, al regular el peso del grado de instrucción de los sujetos mediante un ANOVA de medidas repetidas con una covariante constante.

El ANOVA de medidas repetidas calculado con el índice de interferencia para la condición de palabras de Amenaza Física, puso de manifiesto la presencia de efectos principales significativos para el factor grupo, $F(2,48)=6,94, p<0,005$, y la interacción grupo $x$ tarjeta, $F(2,48)=3,24, p<0,05$; sin embargo, no fue significativo el factor tarjeta, $F(1,48)=2,82$. En el ANOVA de medidas repetidas con una covariante constante (nivel de instrucción) de nuevo resultó significativo el factor grupo, $F(2,47)=5,16, p<0,01$. Atendiendo a los

Tabla 1. Diferencias entre los grupos en los índices de velocidad de la tarea de Stroop

\begin{tabular}{|c|c|c|c|c|}
\hline Tarea de Stroop & $\begin{array}{l}\text { Hipocondría } \\
\text { M (DE) }\end{array}$ & $\begin{array}{c}\text { Trastorno por angustia } \\
M \text { (DE) }\end{array}$ & $\begin{array}{l}\text { Normal } \\
M \text { (DE) }\end{array}$ & $F$ \\
\hline \multicolumn{5}{|l|}{ Indice de Velocidad } \\
\hline $\begin{array}{l}\text { Tarjeta de "Oes" } \\
\text { Tarjeta Neutra } \\
\text { Tarjeta de Amenaza Social } \\
\text { Tarjeta de Amenaza Física } \\
\text { Tarjeta de Colores }\end{array}$ & $\begin{array}{l}1,37(0,33) \\
1,10(0,31) \\
1,11(0,32) \\
1,02(0,31) \\
0,81(0,22)\end{array}$ & $\begin{array}{l}1,43(0,21) \\
1,17(0,20) \\
1,20(0,21) \\
1,08(0,23) \\
0,88(0,16)\end{array}$ & $\begin{array}{l}1,56(0,35) \\
1,34(0,27) \\
1,33(0,20) \\
1,39(0,23) \\
1,01(0,20)\end{array}$ & $\begin{array}{l}1,68 \\
3,69^{*} \text { (b) } \\
3,42^{*}(\mathrm{~b}) \\
9,66^{*}{ }^{*}(\mathrm{~b}, \mathrm{c}) \\
4,65^{\circ} \text { (b) }\end{array}$ \\
\hline
\end{tabular}

Nota: Diferencias significativas: $a=$ hipocondría-trastorno por angustia; $b=$ hipocondrfa-normal; $c=$ trastorno por angustia-normal.

; $p<0,05 ; "$ " $p<0,01 ; \cdots " p<0,005$.

Tabla 2. Diferencias entre los índices de velocidad de la tarea de Stroop en cada grupo

\begin{tabular}{|c|c|c|c|}
\hline Tarea de Stroop & $\begin{array}{c}\text { Hiponcondría } \\
\mathrm{t}\end{array}$ & $\begin{array}{c}\text { Trastorno por angustia } \\
t\end{array}$ & $\begin{array}{c}\text { Normal } \\
t\end{array}$ \\
\hline $\begin{array}{l}\text { Neutra vs. Amenaza Social } \\
\text { Neutra vs. Amenaza Física } \\
\text { Amenaza Social vs. Amenaza Física }\end{array}$ & $\begin{aligned}-0,27 \\
2,55 \\
3,04\end{aligned}$ & $\begin{aligned}-1,03 \\
2,10^{*} \\
3,82^{*}\end{aligned}$ & $\begin{array}{r}0,23 \\
-0,92 \\
-1,63\end{array}$ \\
\hline
\end{tabular}


resultados de la prueba post-hoc de Scheffé, los grupos de hipocondría y trastorno por angustia en comparación con el normal, nombraron más rápidamente las palabras neutras que las de amenaza física.

Respecto al ANOVA de medidas repetidas del índice de contenido (Amenaza Social-Amenaza Física), cabe apuntar que resultaron significativos tanto los factores grupo, $F(2,48)=6,53, p<0,005$, y tarjeta, $F(1,48)=7,71, p<0,01$, como la interacción grupo $\times$ tarjeta, $F(2,48)=8,82, p<0,005$. Al controlar la influencia del nivel de instrucción de los sujetos a través de un ANOVA de medidas repetidas con una covariante constante, el factor grupo continuó siendo significativo, $F(2,47)=4,55$, $p<0,05$. De acuerdo con el contraste $a$ posteriori calculado (prueba de Scheffé), los grupos clínicos en comparación con el normal, nombraron más rápidamente las palabras referidas a amenaza social que las que aluden a amenaza física.

\section{Relación de los índices de la tarea de} Stroop con otras medidas clínicas

En la Tabla 3 se recogen los coeficientes de correlación entre los índices de velocidad de las cinco condiciones de la prueba de Stroop y las estimaciones de las Escalas de Actitud hacia la Enfermedad (IAS),
Cuestionario de Conducta de enfermedad (IBQ), Inventario de Ansiedad Estado-Rasgo (STAI), Inventario de Depresión de Beck (BDI) y la Escala de Hipocondríasis (Hs) del MMPI. Todas las tarjetas correlacionaron significativamente pero en sentido inverso con las diversas medidas clínicas (entre $r=-0,31, p<0,05 ;$ y $r=-0,63$, $p<0,01)$. Las únicas excepciones las constituyeron la tarjeta de "Oes» que no mostró coeficientes de correlación estadísticamente significativos con las IAS y el IBQ. En la Tabla 3 también se pueden consultar los resultados referidos a la relación entre los índices de interferencia de las condiciones de Amenaza Social y Amenaza Física de la prueba de Stroop y los distintos cuestionarios. No se encontraron correlaciones estadísticamente significativas en la tarjeta de Amenaza Social; sin embargo, la tarjeta de Amenaza Física presentó coeficientes de correlación significativos y positivos con todas las medidas (entre $r=0,31, p<0,01 ;$ y $r=0,40, p<0,01$ ) salvo con el STAI-E.

\section{DISCUSIÓN}

A tenor de los resultados obtenidos aplicando la tarea de Stroop podemos afirmar que los pacientes hipocondríacos $\mathrm{y}$ los pacientes con trastorno por angus-

Tabla 3. Relación de los índices de velocidad y de interferencia de la tarea de Stroop con las actitudes hacia la enfermedad y la conducta de enfermedad, la ansiedad, la depresión y los síntomas somáticos, en la muestra global

\begin{tabular}{|c|c|c|c|c|c|c|c|}
\hline \multirow[b]{3}{*}{ Cuestionario } & \multicolumn{7}{|c|}{ Tarea de Stroop } \\
\hline & \multicolumn{5}{|c|}{ Indice de velocidad } & \multicolumn{2}{|c|}{ Ind. de Interferencia } \\
\hline & «Oes» & Neutra & A. Social & A. Física & Color & A. Social & A. Física \\
\hline Es & -1 & $-0,36^{* *}$ & $-0,40^{* *}$ & $-0,55^{\circ *}$ & $-0,39^{* *}$ & 0,01 & $0,37^{* *}$ \\
\hline o de Cond & $-0,21$ & $-0,35^{\star}$ & $-0,37^{* *}$ & $-0,51^{*}$ & $-0,41^{*}$ & $-0,02$ & $0,32^{*}$ \\
\hline 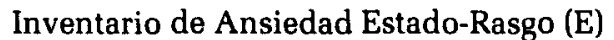 & $-0,38^{* *}$ & $-0,37^{*}$ & $-0,41^{* *}$ & $-0,47^{* *}$ & $-0,47^{*}$ & 0,01 & 0,21 \\
\hline o-Rasgo (R) & $-0,32$ & $-0.33^{\star}$ & $-0,40 * *$ & $-0,54 *$ & $-0,43^{* *}$ & 0,06 & $0,40^{* *}$ \\
\hline Inventario de Depresión de Beck & $-0,39^{* *}$ & $-0,49^{*}$ & $-0,45^{\star *}$ & $-0,63^{* *}$ & $-0,53^{* *}$ & 0,04 & $0,31^{\star}$ \\
\hline Escala de Hipocondríasis (MMPI) & $-0,31^{\circ}$ & $-0,41^{* *}$ & $-0,46^{* *}$ & $-0,59^{* *}$ & $-0,42^{* *}$ & 0,01 & $0,35^{*}$ \\
\hline
\end{tabular}

"p<0,05;" $" p<0,01$. 
tia presentaron tiempos de reacción mayores que los sujetos controles para las palabras de amenaza física. Los pacientes hipocondríacos también mostraron una latencia mayor que los controles normales para las palabras neutras, de amenaza social y de colores, es decir, la lentitud en el procesamiento de la información parece afectar en estos pacientes a todo tipo de material con independencia de su contenido semántico.

Por otra parte, se observó que los dos grupos clínicos, en comparación con el normal, tardaban más tiempo en nombrar los colores de los estímulos de amenaza física que los de los estímulos neutrales y de amenaza social. Además, el análisis intra-grupo reveló que tanto los pacientes hipocondríacos como aquellos con trastorno por angustia, mostraron una latencia mayor para las palabras de amenaza física que para las neutras, no apreciándose este efecto para el material de amenaza social (vs. palabras neutras). Este sesgo en el procesamiento selectivo de la información que describe peligros físicos se siguió manteniendo cuando se controló el posible efecto de la emocionalidad negativa con los estímulos de amenaza social. Así, ambos grupos clínicos tardaron más tiempo en nombrar las palabras de amenaza física que las palabras de amenaza social.

En general, nuestros resultados apoyan la hipótesis de la especificidad del análisis cognitivo de la información amenazante en la hipocondría y el trastorno por angustia. Los pacientes con estos desórdenes procesan preferentemente el material que contiene significados coherentes con el núcleo sintomático que les caracteriza: la preocupación por la enfermedad y la muerte.

De acuerdo con la revisión de la literatura que hemos realizado, no existe ningún estudio que haya analizado las tendencias atencionales de los pacientes hipocondríacos utilizando el paradigma de Stroop.
No disponemos, por tanto, de trabajos que nos sirvan como puntos de referencia para contrastar nuestros resultados.

En cuanto al trastorno por angustia, nuestros datos están en la línea de algunos estudios previos sobre sesgos atencionales en este tipo de muestras clínicas que han utilizado versiones modificadas del paradigma de Stroop. En su formato original con tarjetas diversos estudios han demostrado que en los pacientes con este trastorno se observa un efecto de interferencia al nombrar el color de palabras de contenido amenazante. Ehlers, Margraf, Davies y Roth (1988) utilizando tarjetas compuestas por palabras de amenaza física (p. ej., enfermedad), separación (p. ej., soledad), azoramiento (p. ej., humillación) y neutras o positivas, encontraron que los pacientes con trastorno por angustia así como los sujetos no clínicos con crisis de angustia, en comparación con los sujetos normales, nombraron más lentamente las palabras de amenaza (tanto física como social) que las neutras. Carter et al. (1992) compararon tres grupos de sujetos (trastorno por angustia, trastorno de depresión mayor y normales), utilizando una tarea de Stroop con tarjetas que incluían palabras neutras, de amenaza física, de ansiedad (evaluadas previamente por un grupo de pacientes con trastorno por angustia con agorafobia) y de depresión. En su estudio encontraron que comparados con los controles normales, los pacientes con trastorno por angustia mostraban mayor interferencia para las palabras de amenaza física, mientras que los pacientes deprimidos sólo mostraban una tendencia (que no llegaba a ser significativa) hacia las palabras de depresión. Sin embargo, los pacientes con trastorno por angustia también exhibieron una interferencia mayor para las palabras de depresión que los normales.

Nuestros resultados, a diferencia de los de Ehlers et al. (1988) y Carter et al. 
(1992), sugieren que el sesgo en el procesamiento de la información que exhiben los pacientes con trastorno por angustia no es de carácter general sino específico, esto es, se restringe exclusivamente a los estímulos de amenaza física.

Los trabajos que han utilizado la versión computerizada de esta prueba también han vertido resultados interesantes. McNally et al. (1990) compararon un grupo de pacientes con trastorno por angustia y un grupo control normal compuesto por sujetos con experiencia en el tratamiento de este trastorno psicológico. Los tipos de palabras que utilizaron fueron: palabras de miedo (p. ej., pánico), de sensaciones corporales (p. ej., mareo), de catástrofe (p. ej., muerte) y neutras (p. ej., típico). Estos autores encontraron que el grupo clínico mostraba tiempos de reacción mayores ante todas las modalidades de palabras en comparación con los sujetos controles. También observaron que aunque ambos grupos mostraban más interferencia para las palabras de catástrofe que para las de sensaciones corporales y las de miedo, en el grupo clínico la magnitud de las interferencia para los tres tipos de estímulos amenazantes era mayor que en el normal.

McNally, Riemann, Louro, Lukach y Kim (1992) utilizando estímulos neutrales (una fila de $6 X$ ), positivos (p. ej., felicidad), de miedo (p. ej., terror), de sensaciones corporales (p. ej., mareo) y de catástrofe (p. ej., infarto), observaron que los pacientes con trastorno por angustia tardaban más tiempo en nombrar las palabras de catástrofe que las positivas, pero que mostraban una latencia de respuesta similar para las palabras positivas, de miedo y de sensaciones corporales. Los pacientes con trastorno obsesivocompulsivo mostraban en general tiempos de respuesta más prolongados en comparación con pacientes con trastorno por angustia y sujetos controles normales, y su patrón de interferencia se ase- mejaba al de los pacientes con trastorno por angustia ante palabras que se espera que sean específicas de esta condición.

McNally, Amir, Louro, Lukach, Riemann y Calamari (1994) compararon tres grupos de sujetos (trastorno por angustia, trastorno obsesivo-compulsivo y normal) en un estudio en el que utilizaron palabras de amenaza de pánico (p. ej., miedo), positivas de pánico (p. ej., seguro), de amenaza general (p. ej., soledad) y neutras (p. ej., idéntico). Estos autores encontraron que los pacientes con trastorno por angustia tenían latencias mayores en comparación con los otros dos grupos. Además, este grupo mostró mayor interferencia para las palabras de amenaza de pánico que para las palabras positivas, obteniendo el grupo de pacientes con trastorno obsesivo-compulsivo el patrón opuesto. Por otra parte, y a diferencia de los pacientes de este último grupo, aquellos con trastorno por angustia exhibieron mayor interferencia para las palabras de amenaza (pánico y general) que para las neutras; sin embargo, este grupo no mostró mayor interferencia para las palabras de amenaza de pánico que para las de amenaza general.

En el estudio de Quero, Baños y Botella (1996) participaron pacientes con trastorno por angustia, sujetos normales y psicólogos expertos en el tratamiento del trastorno por angustia, y se utilizaron palabras de amenaza-pánico (p. ej., ambulancia), palabras de amenaza-social (p. ej., tonto) y palabras neutras (p. ej., fotografía). Estas autoras sólo pudieron comprobar la existencia de mayor interferencia para las palabras de amenazapánico en el grupo clínico cuando se consideró en éste únicamente a los pacientes que presentaban agorafobia y se seleccionaron las palabras que mayor significado negativo tenían para los pacientes. En este trabajo también se observó que la evaluación emocional de las palabras no correlacionó con mayores 
latencias de respuesta ni con mayores interferencias, y que los pacientes y los expertos presentaron un patrón de interferencia similar aunque las diferencias intra-grupo fueron mayores en el grupo clínico.

Maidenberg, Chen, Craske, Bohn y Bystritsky (1996) presentaron a pacientes con trastorno por angustia, fobia social y controles normales, palabras de amenaza de pánico (p. ej., mareado), positivas de pánico (p. ej., sano), amenaza social (p. ej., criticado), positivas social (p. ej., admirado), amenaza general (p. ej., violencia), positivas general (p. ej., agradable) y neutras (p. ej., estructura). En este estudio se encontró que el grupo de trastorno por angustia, a diferencia de los otros grupos, necesitó más tiempo para nombrar las palabras de amenaza (pánico, social y general) que para nombrar las palabras neutras; en cambio, en el grupo de fobia social la mayor latencia de respuesta sólo correspondió a las palabras de amenaza social. Asimismo, se observó que el grupo de trastorno por angustia tardó el mismo tiempo en nombrar las palabras positivas de pánico que las neutras. Una tendencia similar se observó en el grupo de fobia social con las palabras positivas social y las neutras.

Como acabamos de exponer, los estudios que han utilizado muestras de pacientes con trastorno por angustia no han encontrado evidencia suficiente que permita concluir que los efectos observados en la tarea de Stroop puedan ser explicados a través de la hipótesis de la familiaridad (la interferencia se debe a la habituación a tratar con esas palabras) ( $p$. ej., Quero et al., 1996) o la hipótesis de la emocionalidad (la interferencia se debe a la carga emocional de las palabras con independencia de si tienen valencia positiva o negativa) (p. ej., Maidenberg et al., 1996). Basándonos en la evidencia existente, aunque reconociendo que ésta no es totalmente concluyente, consideramos legítimo interpretar los efectos de interferencia de la tarea de Stroop que hemos detectado en las muestras clínicas de hipocondría y trastorno por angustia como evidencia de la actuación de un sesgo atencional congruente con la preocupación principal de los sujetos.

Por otra parte, y centrándonos en los datos procedentes del análisis correlacional podemos afirmar que nuestros resultados apuntan hacia la consideración de que los sujetos que presentan mayor interferencia para las palabras de amenaza física son los que exhiben mayor ansiedad-rasgo, un estado de ánimo hipotímico, más molestias corporales, y actitudes y conductas de enfermedad más disfuncionales. En cuanto a los estímulos de amenaza social cabe decir que la interferencia para este tipo de palabras es independiente de estas variables. Estos resultados señalan la existencia de un vínculo entre el sesgo atencional específico hacia los peligros físicos y las preocupaciones hipocondríacas.

En cuanto a la explicación de los mecanismos cognitivos que subyacen a los efectos de interferencia de la prueba de Stroop cabe decir que, además de la tradicional (sesgo atencional), Ruiter y Brosschot (1994) han sugerido una explicación complementaria que contempla la evitación cognitiva. Para ello, estos autores se han basado en los trabajos que señalan que los efectos de interferencia de Stroop en los pacientes con trastorno por angustia no se limitan a las palabras de amenaza física (Carter et al., 1992); en los resultados que apuntan que los sujetos con elevada ansiedad rasgo comparados con los que puntúan bajo en esta medida, muestran mayor interferencia para las palabras positivas que para las neutras, pero sólo se evidencia una tendencia para las palabras de amenaza (vs. palabras neutras) (Mogg y Marden, 1990); y en los datos que señalan que los sujetos «represores» presentan más interferencia 
que los sujetos con nivel elevado de ansiedad rasgo para las palabras de ira, ansiedad y pena (Dawkins y Furnham, 1989). Según Ruiter y Brosschot (1994), los efectos de interferencia constatados en la tarea de Stroop pueden estar reflejando un estilo general de evitación del procesamiento de los estímulos que contienen información emocional. Estos autores no descartan la posibilidad de que tales efectos sean debidos a un sesgo atencional. De hecho, plantean que en la tarea de Stroop «emocional» estarían involucrados ambos procesos: en las primeras etapas actuaría el sesgo atencional y en las finales la evitación cognitiva.

En los últimos años algunos autores (MacLeod y Hagen, 1992; MacLeod y Rutherford, 1992) han sugerido la posibilidad de diferenciar los procesos automáticos de los procesos estratégicos implicados en los sesgos atencionales utilizando distintas modalidades de la tarea de Stroop. La versión enmascarada de la tarea de Stroop se basa en la presentación de estímulos subliminales (que ocurren fuera de la conciencia del sujeto) y permitiría evaluar la atención selectiva que involucra procesos automáticos. La versión no enmascarada de la tarea de Stroop está compuesta por estímulos accesibles a la conciencia y ofrecería la posibilidad de estimar la atención selectiva influida por estrategias de afrontamiento conscientes. En un estudio reciente Thorpe y Salkovskis (1997) han analizado si el sesgo atencional que se observa en los pacientes ansiosos (en concreto en aquellos con fobia a las arañas) es previo o posterior al acceso a la conciencia de la información. Estos autores no encontraron evidencia a favor de un sesgo pre-atencional hacia los estímulos amenazantes medido con la tarea de Stroop enmascarada, sin embargo, si constataron la existencia de un despliegue estratégico de la atención hacia los estímulos amenazantes utilizando la tarea de Stroop no enmascarada. A partir de estos resultados, y frente a la hipótesis de que los sujetos ansiosos presentan un sesgo general y automático en el procesamiento de la información amenazante (Williams, Watt, MacLeod y Mathews, 1988; Mogg, Mathews y Weinman, 1987), Thorpe y Salkovskis (1997) han enfatizado la naturaleza estratégica de los sesgos atencionales de estos sujetos. Según estos autores la persona que se siente amenazada puede orientarse estratégicamente hacia el estímulo amenazante significativo para ella, con el fin de evaluar el peligro y preparar las estrategias necesarias para la reducción de la amenaza. Este proceso se manifiesta en términos de aumento de la latencia hacia el estímulo amenazante solo cuando éste es accesible a la conciencia. Aunque esta hipótesis puede resultar plausible, la evidencia en la que se sustenta es todavía muy limitada, por lo que habrá que esperar que futuros trabajos puedan esclarecer su validez explicativa.

En definitiva, y hasta que estas propuestas alternativas sean suficientemente avaladas, la interpretación más sólida de los resultados obtenidos en el presente estudio consiste en considerarlos como una prueba de la existencia de un sesgo atencional y específico de los pacientes con hipocondría y de los pacientes con trastorno por angustia hacia las señales de amenaza física.

\section{REFERENCIAS}

American Psychiatric Association (1987). Diagnostic and Statistical Manual of Mental Disorders ( ${ }^{r d}$ ed.). Washington, DC: APA (versión española: Barcelona, Masson, 1988).

Barsky, A. J. (1992). Amplification, somatization, and the somatoform disorders. Psychosomatics, 33, 28-34.

Barsky, A. J., Cleary, P. D., Wyshak, G., Spitzer, R., Williams, J., y Klerman, G. (1992). 
A structured diagnostic interview for hypochondriasis: a proposed criterion standard. Journal of Nervous and Mental Diseases, 180, 20-27.

Beck, A. T., Rush, A. J., Shaw, B. F., y Emery, G. (1979). Cognitive Therapy of Depression. New York: Guilford Press (Bilbao, Desclée de Brouwer, 1983).

Carter, C. S., Maddock, R. J., y Magliozzi, J. (1992). Patterns of abnormal processing of emotional information in panic disorder and major depression. Psychopathology, 25, 65-70.

Cioffi, D. (1991). Sensory awareness versus sensory impression: affect and attention interact to produce somatic meaning. Cognition and Emotion, 5, 275-294.

Dawkins, K., y Furnham, A. (1989). The colour naming of emotional words. British Journal of Psychology, 80, 383-389.

Ehlers, A., Margraf, J., Davies, S., y Roth, W. T. (1988). Selective processing of threat cues in subjects with panic attacks. Cognition and Emotion, 2, 201-219.

Foa, E. B., Ilai, D., McCarthy, P. R., Shoyer, B., y Murdock, T. (1993). Information processing in obsessive-compulsive disorder. Cognitive Therapy and Research, 17, 173189.

Hathaway, S. R., y Mckinley, J. C. (1967). Minnesota Multiphasic Personality Inventory. Manual Revised 1967. New York: The Psychological Corporation (Madrid, TEA, 1988).

Hope, D. A., Rapee, R. M., Heimberg, R. G., y Dombeck, M. J. (1990). Representations of the self in social phobia: vulnerability to social threat. Cognitive Therapy and Research, 14, 477-485.

Kellner, R. (1986). Somatization and hypochondriasis. New York: Praeger.

MacLeod, C., y Hagan, R. (1992). Individual differences in the selective processing of threatening information, and emotional responses to a stressful life event. Behaviour Research and Therapy, 30, 151-161.

MacLeod, C., y Rutherford, E. M. (1992). Anxiety and the selective processing of emotional information: mediating roles of awareness, trait and state variables, and personal relevance of stimulus materials. Behaviour Research and Therapy, 30, 479491.
Maidenberg, E., Chen, E., Craske, M., Bohn, P., y Bystritsky, A. (1996). Specificity of attentional bias in panic disorder and social phobia. Journal of Anxiety Disorders, 10, 529-541.

McNally, R. J., Amir, N., Louro, C. E., Lukach, B. M., Riemann, B. C., y Calamari, J. E. (1994). Cognitive processing of idiographic emotional information in panic disorder. Behaviour Research and Therapy, 32, 119122.

McNally, R. J., Riemann, B. C., Louro, C. E., Lukach, B. M., y Kim, E. (1992). Cognitive processing of emotional information in panic disorder. Behaviour Research and Therapy, 30, 143-149.

McNally, R. J., Riemann, B. C., y Kim, E. (1990). Selective processing of threat cues in panic disorder. Behaviour Research and Therapy, 28, 407-412.

Mogg, K., y Marden, B. (1990). Processing of emotional information in anxious subjects. British Journal of Clinical Psychology, 29. 227-229.

Mogg, K., Mathews, A., y Weinman, J. (1987). Memory bias in clinical anxiety. Journal of Abnormal Psychology, 96, 94-98.

Pauli, P., Schwenzer, M., Brody, S., Rau, H., y Birbaumer, N. (1993). Hypochondriacal attitudes, pain sensitivity, and attentional bias. Journal of Psychosomatic Research, 37, 745-752.

Pilowsky, I., y Spence, N. D. (1983). Manual for the Illness Behaviour Questionnaire. Department of Psychiatry, University of Adelaide, Australia.

Quero, S., Baños, R. M., y Botella, C. (1996). Sesgos atencionales y de memoria en el trastorno de angustia. Análisis y Modificación de Conducta, 22, 409-434.

Ruiter, C., y Brosschot, J. F. (1994). The emotional Stroop interference effect in anxiety: attentional bias or cognitive avoidance?. Behaviour Research and Therapy, 32, 315319.

Schmidt, A. J. M., Wolfs-Takens, D. J., Oosterlaan, J., y Van den Hout, M. A. (1994). Psychological mechanisms in hypochondriasis: attention-induced physical symptoms without sensory stimulation. Psychotherapy and Psychosomatics, 61, 117-120. 
Spielberger, C. D, Gorsuch, R L, y Lushene, R. E. (1970). STAI, Manual for the State-Trait Anxiety Inventory (Self Evaluation Questionnaire). Palo Alto, California: Consulting Psychologist Press (Madrid, TEA, 1988). Spitzer, R. L., Williams, J. B. W., Gibbon, M., y First, M. B. (1990). User's Guide for the Structured Clinical Interview for DSM-III$R$. Washington, DC: APP (Barcelona, Trajecte, 1993).

Stroop. J. R. (1935). Studies of interference in serial verbal reactions. Journal of Experimental Psychology, 18, 643-661.
Thorpe, S. J., y Salkovskis, P. M. (1997). Information processing in spider phobics: the Stroop colour naming task may indicate strategic but not automatic attentional bias. Behaviour Research and Therapy, 35, 131. 144.

Warwick, H. M. C., y Salkovskis, P. M. (1990). Hypochondriasis. Behaviour Research and Therapy, 28, 105-117.

Williams, J. M. G., Watts, F. N., MacLeod, C., y Mathews, A. (1988). Cognitive Psychology and Emotional Disorders. Chichester: Wiley. 